\title{
Article
}

\section{Effectiveness and Safety of Antibiotics for Preventing Pneumonia and Improving Outcome after Acute Stroke: Systematic Review and Meta-analysis}

Badve, Monica S, Zhou, Zien, Anderson, Craig and Hackett, Maree Available at http://clok.uclan.ac.uk/23967/

Badve, Monica S, Zhou, Zien, Anderson, Craig and Hackett, Maree ORCID: 0000-0003-1211-9087 (2018) Effectiveness and Safety of Antibiotics for Preventing Pneumonia and Improving Outcome after Acute Stroke: Systematic Review and Meta-analysis. Journal of Stroke and Cerebrovascular Diseases . ISSN 1052-3057

It is advisable to refer to the publisher's version if you intend to cite from the work. http://dx.doi.org/10.1016/j.jstrokecerebrovasdis.2018.07.001

For more information about UCLan's research in this area go to http://www.uclan.ac.uk/researchgroups/ and search for <name of research Group>.

For information about Research generally at UCLan please go to http://www.uclan.ac.uk/research/

All outputs in CLoK are protected by Intellectual Property Rights law, including Copyright law. Copyright, IPR and Moral Rights for the works on this site are retained by the individual authors and/or other copyright owners. Terms and conditions for use of this material are defined in the policies page. 
Title: Effectiveness and safety of antibiotics for preventing pneumonia and improving outcome after acute stroke: systematic review and meta-analysis

Short title: Preventive antibiotics for post-stroke pneumonia

Word count: Abstract: 235 words (max 250 words); Full-text: 2,917 words; excluding title page, abstract, figure legends, and references)

\section{Authors}

Monica S. Badve, ${ }^{1,2,3}$, Zien Zhou, ${ }^{2,4}$, Craig S. Anderson, ${ }^{2,3,5}$, Maree L. Hackett, ${ }^{2,3,6}$ Author affiliations

1. Department of Neurology, The St George hospital, Kogarah, Sydney, Australia

2. Neurological and Mental Health Division, The George Institute for Global Health, Faculty of Medicine, University of New South Wales, Sydney, Australia

3. School of Medicine, The University of Sydney, Camperdown, Sydney, Australia

4. Department of Radiology, Ren Ji Hospital, School of Medicine, Shanghai Jiao Tong University, Shanghai, China

5. The George Institute China at Peking University Health Science Center, Beijing, China

6. Faculty of Health and Wellbeing, The University of Central Lancashire, Preston, United Kingdom

\section{Corresponding author}

Dr Monica S Badve, Ground floor, Prince William Wing, 26 Belgrave street, Kogarah, NSW, Australia 2217. Phone: +61-2-91131111, Fax: +61-2-91132211

Email: monica.badve@health.nsw.gov.au 


\section{ABSTRACT}

Background: Pneumonia is a common complication after stroke which increases morbidity and mortality. This systematic review was conducted to evaluate the efficacy and safety of antibiotics for the prevention of pneumonia after acute stroke.

Methods: Medline, EMBASE and Cochrane databases were searched for randomised controlled trials comparing preventive antibiotics to placebo or no antibiotics after acute stroke. The primary outcome was post-stroke pneumonia. Secondary outcomes were all infections, urinary tract infections, death, dependency, length of hospital stay, and adverse events. Treatment effects were summarised using random-effects meta-analysis.

Results: Six trials (4,111 patients) were eligible for inclusion. The median National Institute of Health Stroke Scale score in included trials ranged from 5 to 16.5. The proportion of dysphagia ranged from $26 \%$ to $100 \%$. Preventive antibiotics were commenced within 48 hours after acute stroke. Compared to control, preventive antibiotics reduced the risk of poststroke pneumonia (RR 0.75, 95\%CI 0.57-0.99), and all infections (RR 0.58, 95\%CI 0.480.69). There was no significant difference in the risks of dependency (RR 0.99, 95\%CI 0.88$1 \cdot 11$ ), or mortality (RR $0 \cdot 96,95 \%$ CI $0 \cdot 78-1 \cdot 19)$ between the preventive antibiotics and control groups. Preventive antibiotics did not increase the risk of elevated liver enzymes (RR 1.20, 95\% CI 0.97-1.49). Preventive antibiotics had uncertain effects on the risks of other adverse events.

Conclusion: Preventive antibiotics reduced the risk of post-stroke pneumonia. However, there is insufficient evidence to currently recommend routine use of preventive antibiotics after acute stroke. 


\section{INTRODUCTION}

Pneumonia is the most common infective complication of acute stroke which occurs in 5\%-26\% of patients with acute stroke (1-3). Post-stroke pneumonia is a pneumonia occurring after acute stroke, usually being hospital-acquired and occurring early (in the first 4 weeks) after acute stroke or late (after 4 weeks)(2). Post-stroke pneumonia can lead to respiratory failure requiring mechanical ventilation, prolonged hospitalisation, and delayed mobilisation $(2,3)$. Thus, post-stroke pneumonia is associated with significant morbidity, mortality and poses an economic burden (4-7). Risk factors associated with post-stroke pneumonia include older age, dysphagia, male gender, stroke severity, pre-admission dependency, coronary artery disease, congestive cardiac failure, and chronic obstructive pulmonary disease $(3,7)$. While lacunar strokes are less likely to predispose patients to developing post-stroke pneumonia compared to larger strokes, stroke-associated immunosuppression can increase the risk of post-stroke pneumonia (8).

In some trials, administering preventive antibiotics has been shown to reduce the risk of post-stroke infection (9-18). However, there is uncertainty as to whether preventive antibiotics reduce post-stroke dependency or mortality, with some studies suggesting improvement, and others showing no difference in outcome compared to standard stroke unit care (9-18). Antibiotic use may lead to complications such as allergic reactions, adverse effects, colonisation with drug-resistant organisms such as methicillin-resistant staphylococcus aureus, or Clostridium difficile diarrhoea $(13,17)$. Therefore, this systematic review was conducted to evaluate the efficacy and safety of prophylactic antibiotics in poststroke pneumonia. 


\section{METHODS}

This systematic review was conducted according to Cochrane methods and reported according to the Preferred Reporting Items for Systematic Reviews and Meta-Analyses (PRISMA) $(19,20)$. The protocol was registered in the International prospective register of systematic reviews (PROSPERO)

\section{www.crd.york.ac.uk/PROSPERO/display_record.asp?ID=CRD42016053133.}

\section{Search strategy and selection criteria}

Medline (Medical Literature Analysis and Retrieval System Online) via Ovid, EMBASE (Excerpta Medical Database) and CENTRAL (Cochrane Central Register of Controlled Trials) were searched from inception to December 2016 (See supplementary files for search strategy). In addition, clinical trial registers, reference lists of relevant review articles, systematic reviews, and treatment guidelines were searched for published and ongoing trials. Missing, incomplete or unpublished data from clinical trials were requested from the respective investigators by email. The following data were extracted using a standardised form: patient demographic details, study design and conduct, rate of outcome events and adverse events. The methodological quality of each study was assessed using the risk of bias assessment tool developed by the Cochrane Bias Methods Group (20). The following eight items were assessed: 1 . random sequence generation; 2 . allocation concealment; 3. blinding of participants, 4 . blinding of investigators 5 , blinding of outcome assessors; 6. incomplete outcome data; 7. selective outcome reporting; 8. any other bias (e.g. insufficient rationale, study design e.g. cluster randomised trials, cross-over trials).

Studies were eligible for inclusion if they: (1) were randomised controlled trials; (2) involved adult patients (age $\geq 18$ years) admitted within 30 days of acute ischemic or hemorrhagic stroke; and (3) compared prophylactic antibiotics for the prevention of 
pneumonia with placebo, no treatment or standard care. There were no language restrictions or study size exclusions. Trials including populations with ischaemic and haemorrhagic strokes were considered.

\section{Outcome measures}

The primary study outcomes were post-stroke pneumonia after acute stroke. Secondary outcomes were all infections, and urinary tract infections after acute stroke, length of hospital stay, dependency and death at discharge, 6 weeks and 12 weeks after acute stroke. The authors' criteria for the diagnosis of pneumonia, all infection and urinary tract infection were accepted. All assessment scales for dependence and stroke severity were accepted, including modified Rankin scale (mRS) score, Barthel Index, Canadian Neurological Scale, European Quality of life scale (See Supplementary files for description of scales) (21-24). Adverse events included clostridium difficile-positive diarrhoea, Methicillin-resistant Staphylococcus aureus (MRSA) colonisation, intensive care unit (ICU) admission, ventilator requirement, elevated hepatic enzymes, acute kidney injury, allergic reactions, drug-induced exanthema, drug-resistant infections and phlebitis.

\section{Data extraction and quality assessment}

Titles and abstracts were screened independently for potentially eligible studies by two investigators (M.S.B and Z.Z). The same authors independently extracted data and assessed risk of bias using the risk of bias assessment tool developed by the Cochrane Bias Methods Group (20).

\section{Data Synthesis and Analysis}

The numbers of dichotomous outcomes were summarized and mean values with standard deviations were collated for continuous outcomes. Risks ratios with $95 \%$ confidence intervals were calculated for dichotomous outcomes. Pooled risk ratios (RR) with 
95\% confidence intervals $(\mathrm{CI})$ were estimated for primary and secondary dichotomous outcomes using the DerSimonian and Laird random-effects model (25). In every case a twosided $\mathrm{p}$-value of $\leq 0.05$ was deemed significant. Q and $\mathrm{I}^{2}$ statistics were used to estimate heterogeneity across studies. An $\mathrm{I}^{2}$ values of $25 \%, 50 \%$ and $75 \%$ were regarded as evidence of low, moderate and high levels of heterogeneity respectively (26).

The potential for small study effects (publication bias) was assessed by testing the funnel plot asymmetry using the Harbord's test.(27) The quality of evidence was summarized according to the Grading of Recommendations Assessment, Development, and Evaluation (GRADE) guidelines (28). All analyses were conducted using Stata/MP (version 14.2, Stat Corp, College Station, Texas).

\section{Role of funding source}

This study had no funding. The corresponding author had access to all data and took final responsibility for submission of this paper. 


\section{RESULTS}

\section{Selection and description of studies}

Six trials including 4,111 stroke patients met the inclusion criteria (Figure 1; Table 1). $(11-13,15-17)$ The mean age of participants ranged from 67 to 78 years. The proportion of male patients ranged from 35\%-57\%. The median baseline National Institute of Health Stroke Scale (NIHSS) score ranged from 5 to 17 (Table1).(29) Duration of follow-up ranged from 12 weeks to 24 weeks $(11-13,16,17)$. One trial which contributed the maximum patients to this study included patients with less stroke severity unlike all the other trials which included moderate to severe strokes (Table 1) (17). One trial only recruited patients with dysphagia which was not a pre-requisite for inclusion in the other trials (13). Data on dysphagia was available in only three trials $(12,13,17)$. In one trial, the proportion of patients with dysphagia was $26-27 \%$ (17). In the other two trials, the proportion of patients with nasogastric tubes ranged from $22-67 \%(12,13)$.

The preventive antibiotics evaluated were ceftriaxone, moxifloxacin, mezlocillin plus sulbactam, levofloxacin and penicillin, in five trials $(11,12,15-17)$. In two trials penicillins were used and these were semisynthetic derivatives of penicillins, and mezlocillin plus sulbactam $(15,16)$. In two trials fluoroquinolones were used, levofloxacin and moxifloxacin $(11,12)$. Ceftriaxone was used in one trial (17). In one trial, a range of antibiotics were used including amoxicillin with or without clarithromycin or with metronidazole or cephalosporins or any suitable antibiotic could be administered (13). In this trial, $78 \%$ of patients received amoxicillin with clavulanic acid with clarithromycin (13). In most trials, preventive antibiotics were administered intravenously or orally and commenced in the first 48 hours post-stroke (Table 1). The duration of treatment ranged from 3 to 7 days $(11-13,16,17)$. All trials excluded patients currently using antibiotics (11-13, 15-17). 
Patients in the control groups received antibiotics if pneumonia or infection was diagnosed. Preventive antibiotics were discontinued, and an appropriate drug initiated in both groups in case of a diagnosed pneumonia or infection in one trial (11). In three trials additional antibiotics were used in case of diagnosed infections in both groups and withdrawal of preventive antibiotics was based on clinical judgement and local antibiotic policy $(13,16$, 17). In one trial, though a regimen of intravenous ceftazidime and tobramycin for pneumonia, intravenous vancomycin for bacteraemia/endocarditis and ciprofloxacin for urinary tract infections, in addition to preventive antibiotics or placebo, was pre-defined, if infections were refractory to therapy, study medication was withdrawn and antibiotics started as per clinical judgement (12). All patients in the trials received standard stroke unit care. The primary meta-analysis was performed excluding two trials $(11,13)$. The STROKE-INF was a cluster randomised trial and the ESPIAS trial did not provide data on post-stroke pneumonia and instead provided data on lower respiratory tract infection (LRTI) $(11,13)$. Both these trials were excluded from the primary analyses due to methodological differences from the other trials $(11,13)$. As the ESPIAS trial criteria for LRTI made it likely that a significant number of people with post-stroke pneumonia were included, this trial data, along with STROKEINF trial data, were included in the sensitivity analyses $(11,13)$. The sensitivity analysis included all the trials (11-13, 15-17).

\section{Risk of bias}

The areas introducing the greatest risk of bias were lack of blinding of patients and investigators to the intervention (Figure 2) (15-17). Patients were not blinded to the intervention in three trials and investigators were not blinded to the intervention in four trials (Figure 2) $(13,15-17)$. Patients, investigators and outcome assessors were not blinded to the intervention in only one trial (15). One trial was graded as unclear risk of bias for 'other 
forms of bias' due to the problems with cluster randomised design, for example recruitment bias, and differential participant recruitment into clusters (28).

\section{Effects of interventions}

\section{Primary outcome measure}

\section{$\underline{\text { Post-stroke pneumonia }}$}

Preventive antibiotics reduced the risk of post-stroke pneumonia in the primary analysis (4 trials; 2757 participants; RR 0.75, 95\% CI 0.57-0.99, $\mathrm{P}=0.04$; Heterogeneity $\chi^{2}=1.83, \mathrm{I}^{2}=0 \%, \mathrm{P}=0.60$; certainty of evidence moderate) (Figure 3; Table 2) (12, 15-17). There was no significant difference in preventing post-stroke pneumonia between the antibiotic and control arms when the STROKE-INF trial was included in the sensitivity analysis (5 trials; 3845 participants; RR 0.85, 95\% CI 0.59-1.2, $\mathrm{P}=0.12$; Heterogeneity $\chi^{2}=7.32 \mathrm{I}^{2}=45.2 \%, \mathrm{P}=0.4$; certainty of evidence moderate) (Figure 4; Table 2$)(12,13,15$ 17). One trial did not specify the number of post-stroke pneumonia patients in the data on lower respiratory tract infections and hence it was excluded from this outcome analysis (11).

\section{Secondary outcome measures}

\section{All infections}

Types of infections included were pneumonia, urinary tract infections, catheterrelated phlebitis, tracheobronchitis, and other infections (not specified in the trial data). Preventive antibiotics were better than control in the primary analysis including the ( 3 trials; 2677 participants; RR 0.58, 95\% CI 0.48-0.69, $\mathrm{P}<0.0001$; Heterogeneity $\chi^{2}=0.27, \mathrm{I}^{2}=0.0 \%$, $\mathrm{P}=0.60$; certainty of evidence high) (Figure 5; Table 2) $(12,16,17)$. In the sensitivity analysis, antibiotics reduced the risk of all infections when compared to control, but with moderate heterogeneity ( 5 trials; 4030 participants; RR 0.67, 95\% CI 0.52-0.85, $\mathrm{P}=0.001$; 
Heterogeneity $\chi^{2}=9.18, \mathrm{I}^{2}=56.4 \%, \mathrm{P}=0.05$; certainty of evidence moderate) (see Supplementary files for forest plot; Table 2) $(11-13,16,17)$.

\section{Urinary tract infections}

Preventive antibiotics were better than control in the primary analysis ( 3 trials; 2677 participants; RR 0.49, 95\% CI 0.26-0.89, $\mathrm{P}=0.02$; Heterogeneity $\chi^{2}=4.80, \mathrm{I}^{2}=58.4 \%, \mathrm{P}=0.09$; certainty of evidence high) (Table 2) $(12,16,17)$. Preventive antibiotics were better than control in the sensitivity analysis (4 trials; 3894 participants; RR 0.42, 95\% CI 0.29-0.62, $\mathrm{P}<0.0001$; Heterogeneity $\chi^{2}=4.85, \mathrm{I}^{2}=38.2 \%, \mathrm{P}=0.18$; certainty of evidence high) (Table 2) $(12,13,16,17)$.

\section{Dependency at 12 weeks}

There was no significant difference between antibiotic use and control for dependency (mRS 3-6) at 12 weeks in the sensitivity analysis including the PASS and STROKE-INF trials (3719 patients) (2 trials; 3719 participants; RR 1.00, 95\% CI 0.93-1.08, $\mathrm{P}=0.91$; Heterogeneity $\chi^{2}=1.9, \mathrm{I}^{2}=47.4 \%, \mathrm{P}=0.16$; certainty of evidence moderate) (Table 2$)(13,17)$. Primary analysis for this outcome was not possible since there was no data from other trials.

\section{Death at 12 weeks}

There was no significant difference between antibiotic use and control for mortality at day 90 in the primary analysis (4 trials; 2733 patients; RR 0.96, 95\% CI 0.78-1.19, $\mathrm{P}=0.75$; Heterogeneity $\chi^{2}=2.45, \mathrm{I}^{2}=0 \%, \mathrm{P}=0.48$; certainty of evidence moderate) $(12,15-17)$, or sensitivity analysis (6 trials; 4050 participants; RR 1.08, 95\% CI 0.90-1.29, $\mathrm{P}=0.31$; Heterogeneity $\chi^{2}=5.86, \mathrm{I}^{2}=14.6 \%, \mathrm{P}=0.32$; certainty of evidence moderate) (Table 2) (11-13, $15-17)$. 


\section{$\underline{\text { Other outcomes }}$}

Meta-analysis was not conducted for the European Quality of life scale, Canadian neurological scale, the Barthel Index, length of stay, dependency and death at discharge and within 6 weeks because of absent or insufficient available data for pooling (22-24).

\section{$\underline{\text { Adverse events }}$}

Preventive antibiotics did not increase the risk of elevated liver enzymes ( 3 trials; 2652 participants; RR $1.20,95 \%$ CI $0.97-1.49, \mathrm{P}=0.08$; Heterogeneity $\chi^{2}=0.34$, $\mathrm{I}^{2}=12.6 \%, \mathrm{P}=0.84$; certainty of evidence moderate $)(12,16,17)$. Preventive antibiotics did not increase the risk of drug resistant infections ( 2 trials; 2591 participants; RR 1.37, 95\%CI $0.45-4.16, \mathrm{P}=0.57$; Heterogeneity $\chi^{2}=0.28, \mathrm{I}^{2}=0.1 \%, \mathrm{P}=0.59$; certainty of evidence low) (12, 13, 17). Preventive antibiotics also did not increase the risk of MRSA infection ( 2 trials; 1296 participants; RR 0.83, 95\%CI 0.39-1.77, $\mathrm{P}=0.63$; Heterogeneity $\chi^{2}=0.0 .69, \mathrm{I}^{2}=0 \%, \mathrm{P}=0.83$; certainty of evidence low) $(12,13)$. Preventive antibiotics did not increase the risk of clostridium difficile diarrhoea (2 trials; 3729 participants; RR 1.12, 95\% CI 0.12-10.35, $\mathrm{P}=0.9$; Heterogeneity $\chi^{2}=1.79, \mathrm{I}^{2}=44.1 \%, \mathrm{P}=0.18$; certainty of evidence low $)(13,17)$. Only one trial provided data on acute kidney injury, gastrointestinal bleeding, ICU admission, phlebitis, ventilator use, allergic reactions, and drug induced exanthema, and hence metaanalysis was not possible $(12,13,16,17)$. 


\section{DISCUSSION}

In this systematic review (4,111 patients), preventive antibiotics reduced the risk of pneumonia (primary analysis), infection, and urinary tract infection after stroke $(11-13,15-$ 17). However, there was no reduction in mortality or improved functional outcome with preventive antibiotics. There was no significantly increased risk of elevated liver enzymes, drug resistant infections, MRSA colonisation, or clostridium difficile diarrhoea with a low to moderate certainty of evidence. There was insufficient data available to determine the impact of antibiotics on other adverse events.

Three previous systematic reviews showed that preventive antibiotics reduced the risk of post-stroke infection $(6,10,30)$. Only one previous systematic review reported poststroke pneumonia as an outcome measure (30). In this review, preventive antibiotics did not reduce the risk of post-stroke pneumonia (30). There was no improvement in functional outcome or reduction in mortality in any of the previous systematic reviews $(10,30,31)$. Significant adverse events were not reported in previous systematic reviews $(10,30,31)$.

The STROKE-INF trial was cluster randomised, making the results difficult to compare to the other randomised trials (13). In the STROKE-INF trial, a range of antibiotics was allowed, and this could have compromised the effectiveness of antibiotics (13). In the STROKE-INF trial, $34 \%$ of the patients in the control group received antibiotics while infections were diagnosed in only $24 \%$ of them suggesting that control group patients were also receiving preventive antibiotics $(13,32)$. This could have confounded the results of the trial $(13,32)$. Being cluster randomised, the STROKE-INF trial could have led to preferential recruitment of patients at risk of post-stroke pneumonia into the preventive antibiotics group, resulting in a negative result for preventive antibiotics (13). However, there was no difference in the baseline characteristics of the two groups in this trial. 
The main issue with the PASS trial (2538 patients) contributing the largest number of patients to this systematic review, was the low rate of post-stroke pneumonia compared to scientific literature, and milder strokes compared to other trials included in this study $(12,13$, 15-17, 33). This may have reduced the effect of preventive antibiotics on improving outcomes. This trial had $83 \%$ weightage in the primary meta-analysis for post-stroke pneumonia (17). The STROKE-INF trial specified that dysphagia was a pre-requisite for recruitment (13). Only up to $27 \%$ patients experienced dysphagia in the PASS trial (17). There was inadequate reporting of dysphagia in the other trials $(11,15,16)$. In two trials, patients were lost to follow-up and this could have caused attrition bias reducing the effect of preventive antibiotics $(12,17)$.

It is possible that post-stroke pneumonia is a respiratory syndrome and a marker of poor outcome, and hence preventive antibiotics have not been shown to improve outcome in this meta-analysis (17). Similarly, it is possible that post-stroke infection is a marker of poor functional outcome (17). Finally, it is likely that stroke unit care has improved so much that preventive antibiotics, in addition, are not superior in improving outcomes from preventing post-stroke pneumonia and post-stroke infections.

The strengths of this study are that it represents a comprehensive overview of the available evidence, with risk of bias assessment, rating certainty of evidence, and inclusion of only randomised controlled trials. We recognise our study has limitations, for the inclusion of trial-level rather than individual patient data which did not allow further analysis according to particular patient characteristics defined by age, sex, stroke severity, or antibiotic type. There was significant clinical heterogeneity in the trials with different antibiotics, variable onset and duration of treatment, and follow-up post-stroke, with inadequate assessment and reporting of adverse events. Although we assessed publication bias using the recommended technique, this test may not have adequate power to distinguish chance from real asymmetry, as there 
were fewer than 10 trials included. While the overall trial quality was fair, there were only six trials, and the open nature of four meant that participants were not blinded in three trials and investigators were not blinded in four trials (Table 2) $(13,15-17)$.

Based on this review, adequately powered double-blinded randomised trials, including moderate to severe acute stroke patients with dysphagia after acute stroke are required to determine whether preventive antibiotics after the onset of acute stroke prevent pneumonia and improve outcome with good safety and cost-effectiveness. There is insufficient evidence to recommend routine provision of antibiotics to prevent post-stroke pneumonia or infection, and uncertainty over the balance of potential benefits and harms of preventive antibiotics. In this study, preventive antibiotics were not superior to standard stroke unit care in improving functional outcomes or reducing mortality. This would indicate that there should be a greater emphasis on stroke unit care for patients with acute stroke to prevent post-stroke pneumonia and improve outcomes in these patients. 
Contributors: Conception and design: Monica Badve, Maree Hackett, Craig Anderson. Literature search and data extraction: Monica Badve, Zien Zhou. Analysis and interpretation of data: Monica Badve, Maree Hackett. Initial drafting of manuscript: Monica Badve. Critical revision of the manuscript for intellectual content: Monica Badve, Maree Hackett, Zien Zhou, Craig Anderson. Final approval of the manuscript: Monica Badve, Zien Zhou, Craig Anderson, Maree Hackett

Conflicts of interests: The authors declare no conflicts of interests.

Acknowledgements: During the completion of this review Zien Zhou held a research grant from Shanghai Health and Family Planning Commission (No. 20144Y0119), Maree Hackett held a National Heart Foundation Future Leader Fellowship (2014-2017, 100034), and Craig Anderson held a National Health and Medical Research Council (NHMRC) Senior Principal Research Fellowship. These sources of funding had no role in this study.

Funding: None 


\section{References}

1. Westendorp WF, Nederkoorn PJ, Vermeij JD, Dijkgraaf MG, van de Beek D. Poststroke infection: a systematic review and meta-analysis. BMC Neurol. 2011;11:110.

2. Teramoto S. Novel preventive and therapuetic strategy for post-stroke pneumonia. Expert Review of Neurotherapeutics. 2009;9(8):1187-200.

3. Finlayson O, Kapral M, Hall R, Asllani E, Selchen D, Saposnik G, et al. Risk factors, inpatient care, and outcomes of pneumonia after ischemic stroke. Neurology. 2011;77(14):1338-45.

4. Wilson RD. Mortality and cost of pneumonia after stroke for different risk groups. J Stroke Cerebrovasc Dis. 2012;21(1):61-7.

5. Koennecke HC, Belz W, Berfelde D, Endres M, Fitzek S, Hamilton F, et al. Factors influencing in-hospital mortality and morbidity in patients treated on a stroke unit. Neurology. 2011;77(10):965-72.

6. van de Beek D, Wijdicks EF, Vermeij FH, de Haan RJ, Prins JM, Spanjaard L, et al. Preventive antibiotics for infections in acute stroke: a systematic review and meta-analysis. Arch Neurol. 2009;66(9):1076-81.

7. Ovbiagele B, Hills NK, Saver JL, Johnston SC, California Acute Stroke Prototype Registry I. Frequency and determinants of pneumonia and urinary tract infection during stroke hospitalization. J Stroke Cerebrovasc Dis. 2006;15(5):209-13.

8. Hug A, Dalpke A, Wieczorek N, Giese T, Lorenz A, Auffarth G, et al. Infarct volume is a major determiner of post-stroke immune cell function and susceptibility to infection. Stroke. 2009;40(10):3226-32.

9. Meisel C, Prass K, Braun J, Victorov I, Wolf T, Megow D, et al. Preventive antibacterial treatment improves the general medical and neurological outcome in a mouse model of stroke. Stroke. 2004;35(1):2-6. 
10. Westendorp WF, Vermeij JD, Vermeij F, Den Hertog HM, Dippel DW, van de Beek D, et al. Antibiotic therapy for preventing infections in patients with acute stroke. Cochrane Database Syst Rev. 2012;1:CD008530.

11. Chamorro A, Horcajada JP, Obach V, Vargas M, Revilla M, Torres F, et al. The Early Systemic Prophylaxis of Infection After Stroke study: a randomized clinical trial. Stroke. 2005;36(7):1495-500.

12. Harms H, Prass K, Meisel C, Klehmet J, Rogge W, Drenckhahn C, et al. Preventive antibacterial therapy in acute ischemic stroke: A randomized controlled trial. PLoS ONE. 2008;3 (5) (no pagination)(e2158).

13. Kalra L, Irshad S, Hodsoll J, Simpson M, Gulliford M, Smithard D, et al. Prophylactic antibiotics after acute stroke for reducing pneumonia in patients with dysphagia (STROKEINF): A prospective, cluster-randomised, open-label, masked endpoint, controlled clinical trial. The Lancet. 2015;386(10006):1835-44.

14. Lampl Y, Boaz M, Gilad R, Lorberboym M, Dabby R, Rapoport A, et al. Minocycline treatment in acute stroke: an open-label, evaluator-blinded study. Neurology. 2007;69(14):1404-10.

15. De Falco FA, Santangelo R, Majello L, Angelone P. Antimicrobial prophylaxis in the management of ischemic stroke.

. Rivista di Neurobiologia. 1998;44(1):63-7.

16. Schwarz S, Al-Shajlawi F, Sick C, Meairs S, Hennerici MG. Effects of prophylactic antibiotic therapy with mezlocillin plus sulbactam on the incidence and height of fever after severe acute ischemic stroke: the Mannheim infection in stroke study (MISS). Stroke. 2008;39(4):1220-7. 
17. Westendorp WF, Vermeij JD, Zock E, Hooijenga IJ, Kruyt ND, Bosboom HJ, et al. The Preventive Antibiotics in Stroke Study (PASS): a pragmatic randomised open-label masked endpoint clinical trial. Lancet. 2015;385(9977):1519-26.

18. Majkowski J, Kunicka J, Szabelska K, Cendrowski W. Prophylactic use of penicillin G and ampicillin in stroke. I. Clinical observations. [Polish]. Neurologia i neurochirurgia polska. $1982 ; 16(4): 261-7$.

19. Liberati A, Altman DG, Tetzlaff J, Mulrow C, Gotzsche PC, Ioannidis JP, et al. The PRISMA statement for reporting systematic reviews and meta-analyses of studies that evaluate health care interventions: explanation and elaboration. Ann Intern Med. 2009;151(4):W65-94.

20. Higgins JPT, Green S. Cochrane Handbook for Systematic Reviews of Interventions Version 5.1.0 [updated March 2011]. The Cochrane Collaboration, 2011. Available from www.handbook.cochrane.org. .

21. van Swieten JC, Koudstaal PJ, Visser MC, Schouten HJ, van Gijn J. Interobserver agreement for the assessment of handicap in stroke patients. Stroke. 1988;19(5):604-7.

22. Mahoney FI, Barthel DW. Functional Evaluation: The Barthel Index. Md State Med J. 1965;14:61-5.

23. Cote R, Hachinski VC, Shurvell BL, Norris JW, Wolfson C. The Canadian Neurological Scale: a preliminary study in acute stroke. Stroke. 1986;17(4):731-7. 24. EuroQol G. EuroQol--a new facility for the measurement of health-related quality of life. Health Policy. 1990;16(3):199-208.

25. DerSimonian R, Laird N. Meta-analysis in clinical trials. Control Clin Trials. 1986;7(3):177-88.

26. Higgins JP, Thompson SG, Deeks JJ, Altman DG. Measuring inconsistency in metaanalyses. BMJ. 2003;327(7414):557-60. 
27. Harbord RM, Egger M, Sterne JA. A modified test for small-study effects in metaanalyses of controlled trials with binary endpoints. Stat Med. 2006;25(20):3443-57.

28. Guyatt GH, Oxman AD, Kunz R, Vist GE, Falck-Ytter Y, Schunemann HJ, et al. What is "quality of evidence" and why is it important to clinicians? BMJ. 2008;336(7651):995-8.

29. Brott T, Adams HP, Jr., Olinger CP, Marler JR, Barsan WG, Biller J, et al. Measurements of acute cerebral infarction: a clinical examination scale. Stroke. 1989;20(7):864-70.

30. Liu L, Xiong XY, Zhang Q, Fan XT, Yang QW. The Efficacy of Prophylactic Antibiotics on Post-Stroke Infections: An Updated Systematic Review and Meta-Analysis. Sci Rep. 2016;6:36656.

31. Van De Beek D, Wijdicks EFM, Vermeij FH, De Haan RJ, Prins JM, Spanjaard L, et al. Preventive antibiotics for infections in acute stroke: A systematic review and metaanalysis. Archives of Neurology. 2009;66(9):1076-81.

32. Meisel A, Smith CJ. Prevention of stroke-associated pneumonia: Where next? The Lancet. 2015;386(10006):1802-4.

33. Kishore A, Vail A, Chamorro A, Garau J, Hopkins S, Di Napoli M, et al. How is stroke-associated pneumonia diagnosed in clinical stroke research? A systematic review of the literature. International Journal of Stroke. 2014;9:46. 
Legends for figures:

Figure 1: PRISMA diagram

Figure 2: Risk of bias assessment of trials included

Figure 3: Forest plot of comparison: Preventive antibiotics versus control after stroke, primary outcome- post-stroke pneumonia (primary analysis)

Figure 4: Forest plot of comparison: Preventive antibiotics versus control after stroke, primary outcome- post-stroke pneumonia (sensitivity analysis)

Figure 5: Forest plot of comparison: Preventive antibiotics versus control after stroke, secondary outcome- all infections (primary analysis)

Supplemental figure (Supplemental files): Preventive antibiotics versus control after strokesecondary outcome- all infections (sensitivity analysis) 